\title{
La perspectiva geográfica en la investigación sobre ordenamiento territorial
}

\author{
Natalia Flórez* \\ Carlos Eduardo Martínez** \\ David Condori***
}

El objetivo principal del presente ensayo, es reseñar los aspectos geográficos más relevantes dentro del proceso de Ordenamiento Territorial (OT) que se ha venido desarrollando en Co lombia. Se exponen inicialmente, algunas aproximaciones conceptuales sobre el Espacio Geográfico y el OT; luego, se mencionan algunos inconvenientes de los procesos de OT hasta ahora efectuados, deteniéndonos a analizar con mayor profundidad en la deficiente o ninguna incorporación del pensamiento geográfico en la formulación de los Planes de Ordenamiento Territorial (POT), para finalmente plantear y desarrollar algunos aspectos que intentan mostrar los posibles aportes de tres de las corrientes de pensamiento filosófico que más han influido en la Geografía Contemporánea (Positivismo, Estructuralismo y Humanismo) y cómo es posible integrar estos enfoques en los procesos de OT.

Aceptado: junio 2003.

* Bióloga, consultora de manejo de Fauna y Flora silvestre, Fundación WIPANA. Estudiante de Maestría en Geografía , EPG , convenio UPTC - IGAC, Bogotá.

** Ingeniero en Recursos Hídricos. Contraloría General de la República- Universidad Central Facultad de Recursos Hídricos y Gestión Ambiental. Estudiante de Maestría en Geografía , EPG, convenio UPTC IGAC, Bogotá.

*** Agrónomo. Centro de Desarrollo - CEDES. Cochabamba, Bolivia. Estudiante de Maestría en Geografía, EPG, convenio UPTC - IGAC, Bogotá. 


\section{Algunas aproximaciones conceptuales. El análisis del Espacio Geográfico}

Antes de ingresar al tema en cuestión, es conveniente aclarar desde el punto de vista conceptual, algunos términos mencionados en este ensayo. Lücke (2003), señala que el empleo empírico de los términos técnicos sin una base de conocimiento claramente delimitada, tiende a crear confusión al darse un proceso de múltiples percepciones sobre un mismo término. Tal confusión afecta la replicabilidad, así como las opciones de verificación, control y monitoreo de los procesos metodológicos desarrollados y empleados en la generación de escenarios de OT

Así mismo Lücke (2003), recalca que es bastante común observar en el lenguaje geográfico cotidiano, el uso indistinto y muchas veces errado de términos como uso del suelo y uso de la tierra; uso actual de la tierra y cobertura; áreas silvestres, áreas de conservación y áreas protegidas; ordenamiento territorial, capacidad de uso de la tierra y uso potencial de la tierra; ecología y ambiente y territorio y espacio geográfico, entre otros. Para comprender el análisis y el proceso metodológico, es entonces relevante tener clara la diferencia que existe entre campos y conceptos que se manejan en las ciencias geográficas con relación al OT.
Olivier Dollfus en su libro «El análisis geográfico»(1978:7-11), inicia por definir el análisis geográfico como el campo de acción de la Geografía, el cual describe los principales elementos considerados en el análisis geográfico, todo ello enmarcado en las dimensiones de tiempo y espacio. Definir qué estudia la Geografía, es una forma de definir los elementos del análisis geográfico:

"La Geografía como ciencia, estudia los modos de organización del espacio terrestre, así como la distribución de las formas y de las poblaciones (en el sentido de colecciones de individuos) sobre la epidermis de la tierra. Su gestión procede de una dialéctica entre la descripción y la explicación, planteando permanentemente cuestiones que se encadenan y que empiezan por dónde, cómo y por qué”. En principio, la Geografía localiza y sitúa aquello que constituye el objeto de investigación, describe y define las formas para luego proceder al análisis de su disposición, su repetición, su similitud y su singularidad. Como indica Darby citado por Dollfus (1978:7), “la Geografía es entonces, una ciencia en la medida en que los elementos percibidos son examinados y medidos e interpreta los hechos desde diversas perspectivas revalorizándolos de acuerdo y en función de la escala de observación".

De acuerdo con la definición de la Geografía como ciencia y del análisis geográfico como método, Dollfus

152 Natalia Flórez, Carlos E. Martínez, David Condori 
plantea separadamente para efectos de explicación y delimitación de grandes ámbitos, la Geografía Humana y la Geografía Física. Sin embargo, en la realidad la definición de Geografía descansa precisamente en el estudio de las interrelaciones entre sí. Hoy día no se puede concebir el estudio del espacio geográfico sin un enfoque sistémico y holístico de esos grandes ámbitos o dimensiones.

\section{Espacio Geográfico, Territorio y Ordenamiento Territorial}

Tratar de definir las diferencias que puedan existir entre los significados de los términos «Espacio Geográfico», «Territorio» y "Ordenamiento Territorial" no es una tarea fácil.

Dollfus, O. (1976:8-10), define el espacio geográfico de la siguiente forma: «es un espacio diferenciado y localizable que se refleja en el paisaje $y$ determinado por sus características morfológicas (caracterización de sus formas, su repetición, similitud y originalidad), estratigráficas y dinámicas (evolución, sus ritmos y umbrales)".

Dollfus cita a Jean Gottmann (1973) para definir el término "territorio", por lo que también es conveniente revisar las consideraciones de éste último en esa definición. El autor hace un análisis de las diferentes percepciones prejuiciadas de diferentes especialistas del concepto de territorio. Situación que hasta la fecha parece prevalecer con relación a este concepto y a otros que se involucran en el análisis geográfico. El ejemplo más reciente, pero que se origina desde alrededor de los años sesenta, es la percepción de que los conceptos de "Uso del Suelo" y "Uso de la Tierra" significan lo mismo. Estas percepciones equivocadas según Lücke, generalmente pueden llevar a conclusiones igualmente equivocadas del análisis geográfico para producir propuestas de OT que no se apegan a la realidad o que distorsionan su aplicabilidad.

Lücke, O. (2003) menciona que el autor (Gottmann), analiza las percepciones de los políticos, los militares, los juristas para concluir en la siguiente definición geográfica: "El territorio aparece como una noción material y espacial en donde se establecen relaciones esenciales entre lo político, lo social y lo natural". En lo político, el autor -agrega Lücke-, hace un extenso análisis de los conceptos de soberanía, seguridad (en el concepto amplio), felicidad, equidad y progreso con relación a determinado territorio. En la citada definición se generaliza el concepto de límite y de capacidad de soporte, al señalar las nociones materiales y espaciales e inicia la diferenciación entre los conceptos de espacio y territorio.

Es entonces oportuno citar aquí, una definición de Ordenamiento Territorial que el Instituto Geográfico Agustín Codazzi (IGAC) sintetiza de forma muy apropiada sobre los conceptos discutidos anteriormente: «El OT es 
una política de estado y un instrumento de planificación que permite una apropiada organización político-administrativa de la Nación y la proyección espacial de las políticas sociales, económicas, ambientales y culturales de la sociedad, garantizando un nivel de vida adecuado para la población y la conservación del ambiente» (IGAC, 1997:39).

Es necesario también señalar que el autor contextualiza la definición, al detallar el OT como política de Estado, que orienta la planeación del desarrollo desde una perspectiva holística, prospectiva, democrática y participativa. Estos son atributos muy importantes que se podrían adicionar a la definición, ya que señalan la necesidad de evitar darle un carácter sectorial, autoritario, impositivo y coercitivo.

A dicha definición según indica Lücke (2003), se le podría especificar más (si se considera una política, como un curso de acción del presente para el futuro) la dimensión de tiempo, al agregarle “...tanto para las generaciones del presente, como para las del futuro".

Dicha definición quedaría entonces de la siguiente manera: «El OT es una política de estado y un instrumento de planificación del desarrollo desde una perspectiva holística, prospectiva, democrática y participativa. Permite una apropiada organización político-administrativa de la Nación y la proyec- ción espacial de las políticas sociales, económicas, ambientales y culturales de la sociedad, garantizando un nivel de vida adecuado para la población y la conservación del ambiente, tanto para las actuales generaciones, como para las del futuro» (Lücke, 2003).

En relación con la planificación del uso de la tierra, Lücke (1986:17-30) desarrolló algunas de esas consideraciones básicas, las cuales son aplicables al OT. Dichas consideraciones son las siguientes:

"El OT no es, ni debe ser una valoración estática y rígida de cómo deben aprovecharse espacialmente los recursos naturales de una unidad territorial, sino debe ser de naturaleza dinámica y flexible para adecuarse a los cambios tecnológicos, necesidades y valoraciones sobre el entorno físico, psicológico y biológico en las que el ser humano se desenvuelve en el tiempo".

"El OT debe considerar el medio económico y social de manera que, en vez de aumentar la desigualdad social y la concentración de la riqueza, responda positivamente a que los recursos sirvan de la mejor forma al mayor número de gente por el mayor tiempo posible, hacia la búsqueda de un desarrollo sostenible".

154 Natalia Flórez, Carlos E. Martínez, David Condori 
"El OT y la aplicación consecuente de sus resultados y recomendaciones, conducen de por sí a la región o al país involucrado a un mayor desarrollo económico".

"La integración de los objetivos de desarrollo con los de conservación en el OT, es la base de trabajo para equipos multidisciplinarios que busquen un mayor desarrollo institucional, que aseguren una base amplia de éxito en la planificación y manejo de los recursos de un país”.

\section{¿Cómo ordenar un territorio que no} se conoce?

Una frase recurrente que se escucha siempre, y que enfatiza mucho en " $\boldsymbol{E} \boldsymbol{l}$ trabajo del Geógrafo en el tercer mundo" Milton Santos (1991), con relación al OT es: ¿cómo ordenar un territorio que no se conoce? Ciertamente, es una pregunta difícil de responder que resulta siendo algo así como un hito de autoreflexión conciencial de quienes "entienden al menos un poco", de lo que pasa aquí y ahora (nuestro territorio), un mundo complejo y variado, lleno de incertidumbres, enigmas, y particularidades de distinta naturaleza, que involucran relaciones desde el campo estrictamente físico hasta del comportamiento humano individual, con una gran variabilidad escalar entre ellas, como se ha ido resaltando anteriormente.
Muchos son los aspectos que han derivado en las críticas a los POT, como instrumentos de planificación de los recursos, tanto naturales como humanos, no solamente a nivel nacional, sino también en el contexto latinoamericano. Las criticas más duras están dirigidas en su carencia de la explicación de fenómenos físicos pero asociados a las relaciones hombre-naturale$z \boldsymbol{a}$, que permitan establecer algunas predicciones (escenarios) en el tiempo y contrarrestar así distintos tipos de degradación ambiental y riesgos de amenazas de vidas humanas.

La literatura clásica con relación al OT y el papel que juega en ella la Geografía como disciplina aplicada, reporta conceptos, ensayos, metodologías e investigaciones adecuadas y adaptadas lógicamente a los países altamente desarrollados. Paradójicamente, no decimos lo mismo de los países del "tercer mundo", quienes observan y conciben ésta misma problemática desde sus propias visiones y experiencias, muy lejos -dicho sea de paso- de las realidades contrastantes de la mayoría de los países "colonizadores", particularmente europeos, tal como hace hincapié Milton Santos en el trabajo anteriormente mencionado, como geógrafo formado en Francia, que vivió en carne propia- ésta realidad actual.

¿En qué situación se encuentra el proceso de Ordenamiento Territorial Colombiano?

En Colombia, el proceso de OT ha tenido una trayectoria relativamente 
corta, marcada por la divergencia de enfoques e interpretaciones corporativas. En realidad, el Ordenamiento se ha trabajado en el país desde hace más de veinte años, pero casi siempre circunscrito al manejo y conservación de los recursos naturales (Andrade y Amaya, 1996).

El OT se desarrolló paralelamente con los procesos de descentralización iniciados en 1986, temas que son ratificados cinco años más tarde por la Constitución de 1991. Sin embargo, como se mencionó anteriormente, existen diferentes interpretaciones del concepto que hace difícil su aplicación. La Constitución Política de Colombia limita el término Ordenamiento Territorial a procesos político-administrativos, mientras que entidades como la Comisión de Ordenamiento Territorial (COT), Planeación Nacional, el IGAC, Oficinas Departamentales de Planeación, conceptualizan el OT de una forma más amplia, al darle cabida a problemas de ocupación del espacio, económicos, sociales, ambientales y culturales pero cada una de acuerdo a sus intereses y funciones.

Más adelante en 1997, la Ley 388 de Desarrollo Territorial presenta una serie de instrumentos de gestión del suelo, los cuales son considerados como mecanismos o herramientas indispensables para realizar operaciones urbanísticas integrales, que permitan agilizar y encauzar el ordenamiento y desarrollo del territorio.
La Ley 388 de 1997, también estableció unos límites de tiempo para la formulación por parte de las Entidades Territoriales, de los Planes de Ordenamiento Territorial, plazos que fueron ampliados posteriormente. Sin embargo, luego de concluidos los plazos, a abril del 2002, sólo el $47 \%$ de los Municipios del país habían logrado aprobar sus POT. El $35 \%$ de los Municipios, todavía se encontraban en proceso de aprobación por las Corporaciones Autónomas Regionales (CAR), identificándose como "cuello de botella" en esta instancia, el componente ambiental en todos los casos. El restante $18 \%$ de los Municipios, no había superado aun para esa fecha, la etapa de formulación de sus planes (figura 1).

Los departamentos más rezagados en el proceso de OT son: Santander, Boyacá, Nariño, Bolívar, Cauca, Tolima, Chocó y Magdalena. Es importante señalar además, que los $\mathrm{Mu}$ nicipios del país que tienen aprobado sus POT's, presentan una serie de deficiencias que tendrán que ser ajustadas necesariamente. En general, los inconvenientes más importantes identificados en los mencionados procesos, han sido los siguientes:

- Ausencia de un marco regulatorio general. La tan esperada Ley Orgánica de Ordenamiento Territorial (LOOT) aún no ha sido promulgada; la misma, tendría por objeto dictar las normas legales para el Ordenamiento del Territorio colombiano; sujetar a las mismas el

156 Natalia Flórez, Carlos E. Martínez, David Condori 


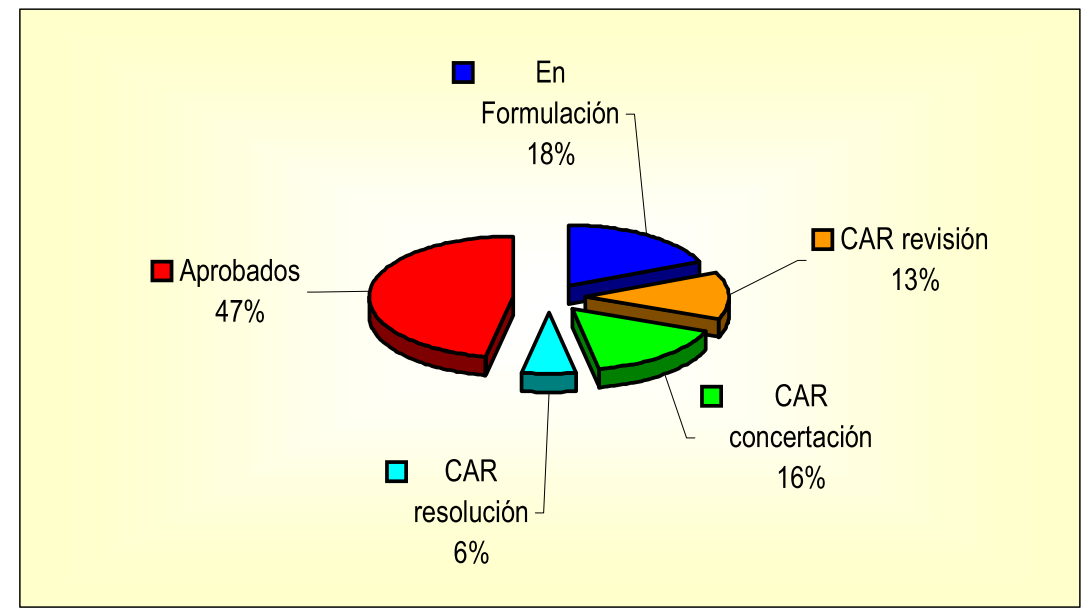

Figura 1. Estado de los POT's en Colombia, hasta abril de 2002.

ejercicio de la actividad legislativa; establecer los principios rectores del ordenamiento; definir el marco institucional e instrumentos para el desarrollo territorial; distribuir competencias entre la Nación y las entidades territoriales y establecer las normas generales para la organización territorial.

- La situación de orden público, que en muchas regiones del país no permitió ni permite el avance normal de estos procesos.

- Insuficiente participación ciudada$\boldsymbol{n} \boldsymbol{a}$, ya que en varias ocasiones el POT se sometió a consideración de la sociedad civil sólo en las etapas finales del proceso.

- Deficiente o ningún aporte del pensamiento geográfico en el proceso de Ordenamiento Territorial.
- Limitada disponibilidad de recursos financieros de los municipios para formular POT integrales, con insuficientes recursos humanos calificados e infraestructura tecnológica adecuada, lo cual deja como resultado POT's con marcadas deficiencias, tanto conceptuales como técnicas.

- En el ámbito institucional, es clara la duplicidad de funciones entre diferentes entidades estatales, por ejemplo el IGAC tiene como funciones desarrollar metodologías de OT y planificación ambiental aplicables a las Entidades Territoriales del país, mientras que el IDEAM tiene funciones similares, relacionadas al ordenamiento ambiental territorial, lo cual lleva a esfuerzos desarticulados y gastos insulsos con poca efectividad real. 
- Los parámetros ambientales establecidos por las CAR a los municipios para el OT, fueron establecidos a partir de información muy fragmentada $e$ insuficiente, particularmente en relación con la caracterización y oferta ambiental de sus jurisdicciones, lo cual reafirma la aseveración de Milton Santos frente al desconocimiento de lo que se ordena.

- La mayoría de los municipios, no contaron con información estructurada y orientada para realizar una adecuada caracterización ambiental y zonificación correspondiente. No se consideró una Línea de Base común, homologable entre las diversas jurisdicciones municipales, departamentales o de territorios naturales estratégicos.

- Existen contradicciones y superposiciones entre la zonificación ecológica y el uso del suelo propuesto por los municipios y las prioridades nacionales, por ejemplo -por citar solo un caso- tenemos las Reservas Forestales Nacionales de la Ley Segunda de 1959 , que se debe o no privilegiar con relación a la Ley de OT.

- Los procesos adelantados en muchas regiones del país, no contaron con el apoyo de los institutos de investigación científica $^{1}$. Esta falta de apoyo, se observó a nivel de las CAR, de los Municipios, y principalmente del nodo central de información ambiental del IDEAM, que a pesar de tener entre sus funciones, el asesoramiento al SINA -en materia de ordenamiento territorial ambiental-, no brindó a su tiempo la información necesaria.

Puede que algunas de estas deficiencias se hayan complementado o que hayan quedado algunas sin señalar, lo importante es recalcar lo planteado al inicio del ensayo, con respecto a no abordar todas las posibles falencias que a la fecha se han presentado en los procesos de OT, lo cual daría para un documento mucho más amplio. El ensayo, únicamente se centra en el análisis del deficiente aporte del pensamiento geográfico en tales procesos, y cómo desde los tres enfoques filosóficos más influyentes en la Geografía Contemporánea, se pueden identificar, integrar y complementar, diferentes corrientes de pensamiento geográfico en el escenario de los procesos de OT.

\section{El Positivismo en el escenario del Ordenamiento Territorial}

No es suficiente sólo definir el OT, sino también plantear algunas consideraciones básicas del papel que debería jugar la Geografía como ciencia aplicada, particularmente de aquella influida por la corriente filosófica del Positivismo clásico y del Neopositivismo de los sesenta, para su aplica-

$\overline{1}$ Estos institutos son el IDEAM, Invemar, Instituto Von Humboldt, Instituto Sinchi y el IIAP. 
ción hoy, ya que las mismas contribuyen a visualizar dicho ordenamiento en su ejecución práctica.

Algunas de las definiciones hechas en la aproximación conceptual sugieren implícitamente y "de hecho" la incorporación fundamentalmente de la Geografía Cuantitativa en el proceso del OT, cuando se dice que el análisis fisiográfico trata de explicar procesos en el espacio para generar escenarios de cambio, -en su sentido más amplio- se aplica uno de los postulados más importantes del Positivismo Lógico de los sesenta. Pero este postulado señalado por Capel (1987:26) en "Las Nuevas Geografias" y otros de la literatura tradicional, queda incompleto ya que el OT en esencia, no es un espacio que se investigue científicamente, es más bien, un instrumento con fuerte componente político-económico-administrativo, por ello, no es posible encontrar de ningún modo, Leyes Científicas de carácter universal, tal cual pregona dicho postulado. Sin embargo, lo rescatable es el esfuerzo que se hace "por lo menos en el papel normativo" (Ley) por explicar procesos e interrelaciones que se dan dentro del territorio. En la realidad, el análisis de la situación de los POT hasta ahora aprobados particularmente en Colombia, arroja resultados lamentablemente discordantes con lo anteriormente señalado.

En este orden de ideas, fieles a los conceptos del proceso de OT, no es posible plantearse hipótesis para explicar fenómenos geográficos que ocurren en el territorio, ni verificarlas, aunque en cierta medida, sea posible aplicar una metodología pero de tipo procedimental, no investigativo. Por tanto, es aquí donde se marca la ruptura de lo que es posible y no, con relación a la adopción de la metodología científica, concordante con el Neopositivismo del siglo XX.

De todas formas, la Geografía Física con relación a lo últimamente señalado (comparado con la Humanística), inclina la balanza a su favor, ya que en el terreno, es decir, "en campo", siempre será posible realizar algunas mediciones, y gracias a los soportes computacionales actuales, logrará con más aproximación, modelar $\mathbf{y}$ comprender procesos geográficos físicos para el OT más rápidamente que antes. Es quizá aquí que la Geografía tiene su mayor aporte actualmente y lo seguirá teniendo seguramente, mientras se supere la crisis de identidad y existencial que ha tenido históricamente hasta hoy, y que Capel (1998:1) muy hábilmente subraya en su ensayo "Una Geografía para el siglo XXI" en el que señala al respecto textualmente:

"Creo que la Geografía debe seguir estudiándose por tres tipos de razones: 1) porque ya existe y es una ciencia con una larga y rica tradición intelectual; 2) porque es una disciplina con un gran valor educativo y formativo; y 3) porque hay problemas que la Geografía, es 
decir los profesionales formados en el campo de la Geografía, pueden ayudar a estudiar y resolver".

\section{El enfoque Estructuralista y el Ordenamiento Territorial}

En términos generales, el enfoque Estructuralista en Geografía busca reconstruir un objeto o fenómeno de manera que salgan a la luz sus leyes de funcionamiento; se trata de identificar, bajo la diversidad de las formas aparentes, aquel pequeño número de principios o esquemas fundamentales que permitan formular unas leyes generales y establecer unas correlaciones estructurales.

Para efectos de OT, el enfoque geográfico Estructuralista aporta elementos importantes para este proceso, dada su concepción holística. Esta característica del Estructuralismo considera los problemas territoriales desde un punto de vista global, ya que intenta involucrar dentro de una perspectiva espacial los aspectos económicos, sociales, culturales y ambientales, usualmente tratados de manera separada.

El OT tiene un enfoque Estructuralista cuando se señala que para potenciar el desarrollo de cada región, es indispensable maximizar el aprovechamiento sostenible de sus ofertas ambientales, reducir la influencia de condiciones o factores limitantes, corregir los problemas, conocer tanto las tendencias de cambio (análisis tendencial) como la naturaleza de las estructuras superiores $e$ inferiores con las cuales se relacionan y definir modelos futuros de desarrollo territorial sostenible (análisis prospectivo).

Giddens (1979) citado por Delgado (2002:219), al respecto señala que "la teoría Estructuralista articula las relaciones entre estructuras sociales $y$ actores sociales, en un esquema conceptual que supere el dualismo que ha caracterizado los discursos de la ciencia social tanto marxista como funcionalista, dado que ambos discursos insisten en la preeminencia del todo social sobre sus partes individuales".

Lo anterior, puede ser una limitante en los procesos de OT ya que deja por fuera el principio participativo $y$ democrático, ya que en el "deber ser" de estos procesos, se busca que el ciudadano participe activamente y forme parte en la toma de decisiones que lo afectan.

\section{El Humanismo en el escenario del Ordenamiento Territorial}

La Geografía Humanística en concepto de citado por Unwin (1975:210280), está relacionada con perspectivas humanistas como el existencialismo y la fenomenología y reconoce que: “...los humanos no existen aparte del mundo en que ellos viven, y dada la diversidad de intereses humanos en dicha organización, existen múltiples mundos, cuyo entendimiento no está sujeto a reglas o leyes generales, ni mucho menos al dominio de la objetividad y de la racionalidad científicas". 
El ecosistema humano considera la semejanza entre las relaciones que mantienen los seres vivos con su ambiente y aquellas que establecen los seres humanos entre ellos mismos. Conceptos como ecosistema, dependencia, singularidad, nicho, medio ambiente y contaminación, resultan adecuados para describir los intercambios culturales, sexuales y afectivos que acontecen en la institución escolar, en el seno de la familia o en la vida social.

Los seres humanos constituimos un ecosistema dotado de un medio ambiente afectivo y simbólico que nos proporciona los elementos necesarios para nuestro sustento emotivo y cultural, de igual manera, es una construcción colectiva en la que participan muchas singularidades, articuladas entre sí para generar soportes culturales y afectivos (Restrepo, 1998).

Tales afirmaciones, provenientes de la Geografía Humanística se identifican con uno de los principios del OT, el cual reconoce la existencia de actores sociales, con intereses, aspiraciones, expectativas y percepciones de los problemas diferentes y en muchos casos marcadamente contradictorias.

De igual manera, la Geografía Humanística propugna por la experiencia interna, por la valoración del conocimiento logrado por la participación más que por la observación, y por el privilegio de la subjetividad sobre la objetividad.
El enfoque humanístico se identifica casi totalmente con el principio participativo que debe considerar el OT, y que como se señaló en las deficiencias de los POT, es una de las mayores fallas que hasta ahora se han presentado en los procesos realizados en el país, no sólo en cuanto a OT sino en muchas otras actividades, que requieren la participación ciudadana.

Dado que no se busca formular un POT para cada individuo, se deben buscar los mecanismos adecuados de participación para considerar los intereses de todos los grupos de actores, para lo cual se deben generar procesos participativos que consideren los tres ejes fundamentales de un proceso participativo efectivo: 1) la capacidad de convocatoria (reconociendo a los otros), 2) el proceso comunicativo (entendiéndose con los otros) y 3) la concertación (construcción de decisiones). Además debe considerarse de alguna manera el análisis de las estructuras sociales, que tenga en cuenta las relaciones, dependencias y singularidades entre individuos, comunidades y lugares.

\section{Conclusiones}

No bastan las "intenciones" de revertir la situación descrita en este ensayo con relación al Ordenamiento Territorial. Las tremendas particularidades de tipo social, económico, físico y cultural de los territorios "colonizados" como son particularmente los latinoamericanos, hace muchas veces ver la inviabilidad 
de su aplicación. A ello se suma sin duda, otros factores como los "políticos", de los cuales en ultima instancia, depende la aplicación del OT, como política de Estado e instrumento de planificación; quienes generalmente no tienen la visión técnica que es necesario tener en estos casos, mucho menos actitudes conservacionistas y sobre todo "humanas" y sociales reales.

Estamos de acuerdo con Dollfus (1976), quien hace una contribución significativa al concluir en su libro lo siguiente: "En el espacio geográfico, la ordenación del territorio es la impresión de una política económica con sus consecuencias sociales, pero es más bien una toma de conciencia, por parte de sus ocupantes, del hecho de que son los depositarios y los avaladores de un patrimonio que es conveniente utilizar del mejor modo posible para las necesidades del momento, al mismo tiempo que lo preparan para las necesidades del futuro".

La formulación de los POT, como estrategia para mejorar la adecuada administración y manejo de los recursos naturales de una región, deberá contemplar el aporte del discurso geográfico, que abarque la amplia diversidad de interacciones y particularidades que suceden en los territorios.
Finalmente, para la formulación de los POT es necesario concebir una jerarquía de criterios o lineamientos que ayuden a priorizar las necesidades del territorio y sus habitantes acorde a sus realidades históricas, actuales y tendencias particulares. En este contexto, el Estado deberá darse a la tarea de establecer políticas de administración y manejo, basados en una metodología que estudie de manera lógica y sistémica, las interdependencias y particularidades, apoyados por los elementos conceptuales de las actuales corrientes del pensamiento geográfico. El reto para el OT es la búsqueda de un enfoque o enfoques que no desconozcan la interiorización de cada uno de los individuos y sus expectativas, lo mismo que las relaciones que se dan en las diferentes estructuras.

Sobre la base de las conclusiones se podría sugerir que la diferencia entre espacio geográfico y territorio estriba básicamente en que: en determinado espacio que está organizado, que es diferenciado, georeferenciado y que se refleja en el paisaje; para que exista el Ordenamiento del Territorio, se define o se imprime una política nacional que cumpla con los requisitos establecidos en la definición de Dollfus. En tal sentido, el Ordenamiento Territorial, requiere de un enfoque integral acorde con la realidad social, ambiental, económica, política y cultural de los territorios. 


\section{Bibliografía}

ANDRADE, A. y AMAYA, M. El Ordenamiento Territorial: Política y Plan, Revista SIGPAFC, $N^{\circ} 10-11$, Bogotá D.C. En: Instituto Geográfico Agustín Codazzi, Bases conceptuales y guía metodológica para la formulación del Plan de Ordenamiento Territorial Ambiental, Bogotá D.C, 1997, p. 30.

CAPEL, Horacio y URTEAGA Luis. Las nuevas geografías. Salvat Editores S.A., Barcelona, 1987, p. 26.

CAPEL, Horacio. Una Geografía para el siglo XXI. Scripta Nova. Revista Electrónica de Geografía y Ciencias Sociales. N\&ordm; 19, 15 de abril de 1998. Universidad de Barcelona. [ISSN 1138-9788]. Available from World Wide Web: <http://www.ub.es/geocrit/ menu.htm >.

DELGADO, Ovidio. Debates sobre el espacio en la geografía contemporánea. Universidad Nacional de Colombia, Bogotá D.C., 2002. 239 p.

DOLLFUS, Olivier. El espacio geográfico, col “QQué sé?”, num. 111. Editorial OikosTau, Vilassar de Mar - Barcelona, 1976. p. 8-10.

DOLLFUS, Olivier. El análisis geográfico, col “Qué sé?”, num. 118. Editorial OikosTau, Vilassar de Mar - Barcelona, 1978. p. 7-11.

INSTITUTO GEOGRAFICO AGUSTIN CODAZZI. Bases conceptuales y guía metodológica para la formulación del Plan de Ordenamiento Territorial Departamental. Ed. Linotopia Bolívar, Bogotá D.C., 1997. p. 39.

LÜCKE, Oscar. Consideraciones Básicas Sobre la Aplicación de Metodologías de Análisis en la Planificación del Uso de la Tierra y la Toma de Decisiones. Centro Agronómico Tropical de Investigación y Enseñanza (CATIE) - Programa de Manejo de Cuencas Hidrográficas. Turrialba, Costa Rica, 1986. p. 17-30. 
LÜCKE, Oscar. Base conceptual y metodología para los escenarios de Ordenamiento Territorial. Consultor Proyecto SINADES ATN/SF/4717-CR, Costa Rica, 2003. Available from World Wide Web: < http://www.mideplan.go.cr/sinades/Proyecto_SINADES/ sostenibilidad/armonizacion/index-2.html $>$.

RESTREPO, Luis. Ecología humana, Ed. San Pablo, Bogotá D.C., 1998. 100 p.

SANTOS, Milton. El trabajo del Geógrafo en el tercer mundo. Sao Paulo: Editora Hucitec, 1991. $80 \mathrm{p}$.

164 Natalia Flórez, Carlos E. Martínez, David Condori 